Pacific Journal of Mathematics

SOME PROPERTIES OF THE CHEBYSHEV METHOD 


\title{
SOME PROPERTIES OF THE CHEBYSHEV METHOD
}

\author{
Matthew LiU AND B. E. RhoAdes
}

\begin{abstract}
Several properties of the Chebyshev method of summability, defined by G. G. Bilodeau, are investigated. Specifically, it is shown that the Chebyshev method is translative and is a Gronwall method. It is shown that the de Vallee Poussin method is stronger than the Chebyshev method, and that the Chebyshev method is not stronger than the $(C, 1)$ method. The final result shows that the Chebyshev method exhibits the Gibbs phenomenon.
\end{abstract}

Let $\Sigma(-1)^{i} u_{i}$ be an alternating series with partial sums $s_{n}=$ $\sum_{i=0}^{n}(-1)^{i} u_{i}$. Define a sequence of polynomials $\left\{P_{n}(t)\right\}$ by $P_{n}(t)=$ $\sum_{k=0}^{n} a_{n k} t^{k}, P_{n}(1)=1, n=0,1,2, \cdots$. The series $\Sigma(-1)^{i} u_{i}$ will be called summable $\left(P_{n}\right)$ to the value $s$ if $\lim \sigma\left(P_{n}\right)=s$, where $\sigma\left(P_{n}\right)=$ $\sum_{l k=0}^{n} a_{n k} s_{k}$. Bilodeau [1] considered the following question. What are sufficient conditions on $P_{n}$ for $\sigma\left(P_{n}\right)$ to speed up the rate of convergence of a convergent sequence $\left\{s_{n}\right\}$ ? For sequences $\left\{u_{n}\right\}$ which are moment sequences, i.e., $u_{n}$ has the representation $u_{n}=\int_{0}^{1} t_{n} d \alpha(t)$, where $\alpha(t) \in B V[0,1]$, he obtains the estimate $\left|\sigma\left(P_{n}\right)-s\right| /\left|r_{n}\right| \leqq$ $\left(\mu_{n} /\left|r_{n}\right|\right) \int_{0}^{1} t(1+t)^{-1}|d \alpha(t)|$, where $s=\sum_{i=0}^{\infty}(-1)^{i} u_{i}, \quad r_{n}=s_{n}-s$, and $\mu_{n}=\max _{0 \leqq t \leqq 1}\left|P_{n}(-t)\right|$. Adopting $\mu_{n}$ as a measure of the value of the method $\sigma\left(P_{n}\right)$, the most desirable sequence of polynomials will be those for which $\mu_{n}$ is a minimum, subject to the constraint $P_{n}(1)=1$ for each $n$. The Chebyshev polynomials, defined by $T_{n}(x)=\cos n x, n=0,1,2, \cdots, x=\cos \theta$, form the best approximation to the zero function over the interval $[-1,1]$. When translated to $[0,1]$ they give $P_{n}(t)=T_{n}(1+2 t) / T_{n}(3)$ as the best polynomials to minimize $\mu_{n}$, where

$$
T_{n}(x)=\left[\left(x+\sqrt{x^{2}-1}\right)^{n}+\left(x-\sqrt{x^{2}-1}\right)^{n}\right] / 2,
$$

and

$$
T_{n}(3)=\left(\alpha^{n}+\alpha^{-n}\right) / 2, \alpha=3+\sqrt{8} \approx 5.828 .
$$

The infinite matrix $A=\left(a_{n k}\right)$, associated with these polynomials, has entries

$$
a_{n k}=\left\{\begin{array}{l}
1 / T_{n}(3), \quad k=0 \\
\frac{\frac{2}{2 k-1}_{T_{n}^{(3)}}\left[2\left(\begin{array}{c}
n+k \\
n
\end{array}\right)-\left(\begin{array}{c}
n+k-1 \\
n-k
\end{array}\right)\right], 0<k \leqq n}{0, \quad k>n .} .
\end{array}\right.
$$


Bilodeau calls the associated summability method the Chebyshev or $\sigma$-method.

We begin by establishing some properties of the maximal entry in each row of $\sigma$.

Lemma 1. For each positive integer $n>2$, there exists an integer $p$ such that

$$
\begin{aligned}
& a_{n k}<a_{n, k+1} \quad \text { for } 0 \leqq k<p \\
& a_{n k} \geqq a_{n, k+1} \quad \text { for } p \geqq k<n .
\end{aligned}
$$

Proof. For $0<k \leqq n$ we may write

$$
a_{n k}=\frac{2^{2 k-1} n}{k T_{n}(3)}\left(\begin{array}{c}
n+k-1 \\
n-k
\end{array}\right),
$$

so that $a_{n k} / a_{n, k+1}=(k+1)(2 k+1) / 2\left(n^{2}-k^{2}\right)$. Treating $k$ as a continuous variable and differentiating with respect to $k$, it follows that $a_{n k} /$ $a_{n, k+1}$ is increasing in $k$. The proof is completed by noting that $a_{n 0}<a_{n 1}<a_{n 2}$ and $a_{n, n-1}>a_{n n}$ for each $n>2$.

Lemma 2. For each $n, p=\left[x_{0}\right]$ where $x_{0}=\left(-3+\left(32 n^{2}-7\right)^{1 / 2}\right) / 8$.

Proof. Since $a_{n 1}<a_{n 2}$ and $a_{n, n-1}>a_{n n}$, there exists a real positive number $x_{0}$ such that $a_{n x_{0}}=a_{n, x_{0}+1}$ which implies $2 x_{0}^{2}+3 x_{0}+1=$ $2 n^{2}-2 x_{0}^{2}$. Since $x_{0}$ is positive, $x_{0}=\left(-3+\left(32 n^{2}-7\right)^{1 / 2}\right) / 8$.

Lemma 3. For each $n>6, p=\left[x_{0}\right]>n / 2$.

It is sufficient to show that $x_{0}-1 \geqq n / 2$; i.e., $8\left(2 n^{2}-11 n-16\right) \geqq$ 0 , for $n>6$. With $g(n)=2 n^{2}-11 n-16$ we have $g^{\prime}(n)>0$ for $n>11 / 4$, hence $g$ is increasing for $n>11 / 4$, and $g$ is positive for $n>6$ and $n$ an integer.

Lemma 4. With $p$ and $a_{n p}$ as defined in Lemmas 2 and 3, $\lim _{n} a_{n p}=0$.

From (3), and Stirling's formula,

$$
\begin{aligned}
a_{n p} & =\frac{n 2^{2 p-1} \Gamma(n+p)}{P T_{n}(3) \Gamma(n-p+1) \Gamma(2 p)} \\
(4) & \sim \frac{n 2^{2 p-1}(n+p-1)^{n+p-1} e^{-(n+p-1)}(2 \pi(n+p-1))^{1 / 2}}{p \alpha^{n}(n-p)^{n-p} e^{-(n-p)}(2 \pi(n-p))^{1 / 2}(2 p-1)^{2 p-1} e^{-(2 p-1)}(2 \pi(2 p-1))^{1 / 2}} \\
& =\frac{1}{2 \sqrt{\pi}} \frac{\left(p-\frac{1}{2}\right)^{1 / 2}}{p} \frac{n}{((n+p-1)(n-p))^{1 / 2}}\left(\frac{n+p-1}{\alpha(n-p)}\right)^{n-p}\left(\frac{n+p-1}{\sqrt{\alpha}\left(p-\frac{1}{2}\right)}\right)^{2 p} .
\end{aligned}
$$


Both $\left((n+p-1) / \alpha(n-p)^{n-p}\right)$ and $\left((n+p-1) / V \bar{\alpha}(p-1 / 2)^{2 p}\right)$ are bounded above. Therefore $\lim _{n} a_{n p}=0$.

Cooke [3, p. 119] shows that a necessary and sufficient condition for a regular matrix to be absolutely translative for all bounded sequences $\left\{z_{n}\right\}$ is that the matrix $A$ satisfies $\lim _{n} \sum_{k=0}^{\infty}\left|a_{n k}-a_{n, k+1}\right|=0$.

THEOREM 1. The $\sigma$-method is absolutely translative for all bounded sequences.

Proof. Bilodeau [1, p. 296] has shown that the $\sigma$-method is regular. From Lemma 1,

$$
\begin{aligned}
\sum_{k=0}^{\infty} \mid a_{n k}- & a_{n, k+1} \mid \\
& =\sum_{k=1}^{p-1}\left(a_{n, k+1}-a_{n k}\right)+\sum_{k=p}^{n}\left(a_{n k}-a_{n, k+1}\right) \\
& =2 a_{n p}-a_{n 0} .
\end{aligned}
$$

The regularity of $A$ implies that $\lim _{n} a_{n 0}=0$, and the result follows from Lemma 4.

For unbounded sequences, we consider the class of sequences $\left\{z_{n}\right\}$ satisfying $\left|z_{k}\right| \leqq \theta_{k}$ ( $\theta_{k}$ real, positive, and increasing), where $\sum_{k=0}^{\infty} a_{n k} \theta_{k+1}, \quad \sum_{k=0}^{\infty} a_{n, k+1} \theta_{k+1}$, and $\rho_{n}=\sum_{k=0}^{\infty}\left|\left(a_{n k}-a_{n, k+1}\right) \theta_{k+1}\right|$ exist for each $n$. Cooke [3, p. 119] shows that a necessary and sufficient condition for a regular matrix to be absolutely translative for all (unbounded) $\left\{z_{n}\right\}$ satisfying $\left|z_{k}\right| \leqq \theta_{k}$ together with conditions stated above, is that $\lim _{n} \rho_{n}=0$.

THEOREM 2. The $\sigma$-method is absolutely translative for all (unbounded) sequences $\left\{z_{n}\right\}$ such that $z_{k}=o(\sqrt{k})$. This result is best possible.

With $\left|\boldsymbol{z}_{n}\right|=\theta_{n}$, and using Lemma 1,

$$
\begin{aligned}
\rho_{n}=\sum_{k=0}^{p-1}\left(a_{n, k+1}-a_{n k}\right) \theta_{k+1}+\sum_{k=p}^{n}\left(a_{n k}-a_{n, k+1}\right) \theta_{k+1} \\
\quad \leqq \theta_{p-1} \sum_{k=1}^{p-1}\left(a_{n, k+1}-a_{n k}\right)+\theta_{n} \sum_{k=p}^{n}\left(a_{n k}-a_{n, k+1}\right) \\
\leqq \theta_{n}\left(a_{n p}-a_{n 0}+a_{n p}-0\right)=0\left(\sqrt{n)}\left(2 a_{n p}-a_{n 0}\right) .\right.
\end{aligned}
$$

It will be sufficient to show that $\overline{\lim }_{n} 2 \sqrt{n a_{n p}}$ is finite. But this follows immediately from (4), since $\lim _{n}(n(p-1 / 2))^{1 / 2} / p=2^{1 / 4}$, and the remaining limits have already been shown to be finite.

To show that the result is best possible we shall replace $o(\sqrt{k})$ 
by $\sqrt{k}$ and verify that $\rho_{n}$ does not tend to zero.

From (5), $\rho_{n} \geqq \sqrt{p} \sum_{k=p}^{n}\left(a_{n k}-a_{n, k+1}\right)=\sqrt{p} a_{n p}$, which does not tend to zero.

Direct calculations verify that $\sigma$ is not a weighted mean, Nörlund, Hausdorff, or generalized Hausdorff method.

Gronwall [4, p. 102] defined a general class of summability methods, each member of which involves a pair of analytic functions $f$ and $g$. Specifically, the $(f, g)$-transform of a given series $\sum_{k=0}^{\infty} u_{k}$ is the sequence $\left\{U_{n}\right\}$ defined implicitly by the formal power series identity

$$
g(w) \sum_{n=0}^{\infty} u_{n}[f(w)]^{n}=\sum_{n=0}^{\infty} b_{n} U_{n} w^{n},
$$

where $f$ and $g$ satisfy the following properties. Let $\Delta=\{w|| w \mid<$ $1\}$. The function $z=f(w)$ is analytic in $\bar{\Delta}-\{1\}$, continuous and $1-1$ in $\bar{\Delta}$, with $f(0)=0, f(1)=1$, and $|f(w)|<1$ for $w \in \Delta$. Moreover, $w=f^{-1}(z)$ has the representation $w=1-(1-z)^{\lambda}\left[a+a_{1}(1-\right.$ $z)+\cdots]$, where $\lambda \geqq 1, a>0$, and the quantity in brackets is a power series in $1-z$ with a positive radius of convergence. The function $g$ satisfies $g(w) \neq 0$ for $w \in \Delta$ and has the form $g(w)=$ $(1-w)^{-\delta}+\gamma(w)$ for some $\delta>0$, where $\gamma(w)$ is analytic in $\bar{\Delta}$. Also $g(w)=\sum_{n=0}^{\infty} b_{n} w^{n}$, with $b_{n} \neq 0$ for each $n$. The series $\sum_{k=0}^{\infty} u_{k}$ is said to be $(f, g)$-summable to $s$ if $\lim U_{n}=s$.

Examples of $(f, g)$-methods are the Cesàro methods of order $k,(C, k)$, for $k$ real and greater than $-1 ;(E, \beta)$ (Euler-Knopp) for $0<\beta \leqq 1$; de la Vallée Poussin summability $(V)$; a generalized $(V)$ summability $(V k)$, introduced by Gronwall; and a method of summation of Obrechkoff. We will now show that the Chebyshev method is also a Gronwall method.

Writing $s_{n}=\sum_{k=0}^{n} u_{k}$, the $(f, g)$-method can be expressed as a sequence to sequence method by rewriting (6) in the form

$$
g(w)[1-f(w)] \sum_{n=0}^{\infty} s_{n}[f(w)]^{n}=\sum_{n=0}^{\infty} b_{n} U_{n} w^{n} .
$$

Using (7), $(f, g)$ can be expressed as a triangular matrix transformation of the form $U_{n}=\sum_{k=0}^{n} a_{n k} s_{k}$, with $a_{n k}=\gamma_{n k} / b_{n}$, where $\gamma_{n k}$ is defined by

$$
[1-f(w)] g(w)[f(w)]^{k}=\sum_{n=k}^{\infty} \gamma_{n k} w^{k}
$$

(See, for example, the discussion on page 40 of [2], where the roles of $\gamma_{n k}$ and $a_{n k}$ have been interchanged.) From (8) it follows that 


$$
a_{n n}=\left[f^{\prime}(0)\right]^{n} / b_{n}, \quad n \geqq 0
$$

Theorem 3. The Chebyshev method is a Gronwall method with $f(w)=w(\alpha-1)^{2} /(\alpha-w)^{2}, \quad g(w)=(1-w)^{-1}+\gamma(w), \quad$ and $\quad \gamma(w)=w /$ $\left(\alpha^{2}-w\right)$, where $\alpha=3+\sqrt{8 \text {. }}$

Proof. If (6) is a Gronwall method, then, from (8) with $k=0$ and (2),

$$
[1-f(w)] g(w)=\sum_{n=0}^{\infty} b_{n} a_{n 0} w^{n}=\sum_{n=0}^{\infty} b_{n} w^{n} / T_{n}(3) .
$$

Thus

$$
\begin{aligned}
& f(w)=1-[g(w)]^{-1} \sum_{n=0}^{\infty} b_{n} w^{n} / T_{n}(3), \\
& f^{\prime}(w)=\left[g^{\prime}(w) / g^{2}(w)\right] \sum_{n=0}^{\infty} b_{n} w^{n} / T_{n}(3)-[g(w)]^{-1} \sum_{n=1}^{\infty} n b_{n} w^{n-1} / T_{n}
\end{aligned}
$$

and $f^{\prime}(0)=\left[g^{\prime}(0) / g^{2}(0)\right]\left(b_{0} / T_{0}(3)\right)-b_{1} / g(0) T_{1}(3)=2 b_{1} / 3 b_{0}$, since $T_{0}(3)=1$ and $T_{1}(3)=3$.

From (9) and (3),

$$
b_{n}=\left(2 b_{1} / 3 b_{0}\right)^{n} T_{n}(3) / 2^{2 n-1}=\left(b_{1} / 6 b_{0}\right)^{n}\left(\alpha^{n}+\alpha^{-n}\right) .
$$

In particular, $b_{1}=b_{1} / b_{0}$, which implies $b_{0}=1$, since each $b_{n} \neq 0$. One can also deduce that $b_{0}=1$ from $(9)$, since $a_{00}=1$.

Thus

$$
\begin{aligned}
g(w) & =1+\sum_{n=1}^{\infty} b_{n} w^{n} \\
& =1+\sum_{n=1}^{\infty}\left[\left(b_{1} \alpha w / 6\right)^{n}+\left(b_{1} w / 6 \alpha\right)^{n}\right] \\
& =1+\frac{b_{1} \alpha w}{6-b_{1} \alpha w}+\frac{b_{1} w}{6 \alpha-b_{1} w} \\
& =\frac{6}{6-b_{1} \alpha w}+\frac{b_{1} w}{6 \alpha-b_{1} w} .
\end{aligned}
$$

For $g$ to have the required form choose $b_{1}=6 / \alpha$.

From (10), and (11), with $b_{1}=6 / \alpha$,

$$
\begin{aligned}
f(w) & =1-[g(w)]^{-1}\left[1+\sum_{n=1}^{\infty} 2(w / \alpha)^{n}\right] \\
& =1-[g(w)]^{-1}\left[1+\frac{2 w}{\alpha-w}\right] \\
& =1-\frac{(\alpha+w)}{\alpha-w} \cdot \frac{(1-w)\left(\alpha^{2}-w\right)}{\left(\alpha^{2}-w^{2}\right)} \\
& =1-\frac{(1-w)\left(\alpha^{2}-w\right)}{(\alpha-w)^{2}}=\frac{w(\alpha-1)^{2}}{(\alpha-w)^{2}} .
\end{aligned}
$$


We now show that $f$ is a $1-1$ selfmapping of $\Delta$. If $f\left(w_{1}\right)=$ $f\left(w_{2}\right)$, i.e.,

$$
\frac{w_{1}(\alpha-1)^{2}}{\left(\alpha-w_{1}\right)^{2}}=\frac{w_{2}(\alpha-2)^{2}}{\left(\alpha-w_{2}\right)^{2}}
$$

then $\left(w_{1}-w_{2}\right)\left(\alpha^{2}-w_{1} w_{2}\right)=0$. Since $w_{1}, w_{2} \in \Delta, w_{1} w_{2} \neq \alpha^{2}$, so $w_{1}=w_{2}$. By the Maximum Modules Theorem, it is sufficient to show that $|f(w)| \leqq 1$ for $w=e^{i \theta} . \quad\left|f\left(e^{i \theta}\right)\right|=(\alpha-1)^{2}\left(\alpha^{2}-2 \cos \theta+1\right) \leqq 1$.

We now verify that $w=f^{-1}(z)$ is regular on $\bar{\Delta}-\Delta$, except possibly at $z=1$, and that $0 \in \Delta . f^{-1}$ is regular except at $z=0$, so now we must show

$$
\min _{0 \leqq \theta<2 \pi}\left|f\left(e^{i \theta}\right)\right| \geqq \delta>0 \text {. }
$$

$\left|f\left(e^{i \theta}\right)\right|=(\alpha-1)^{2} / T(\theta)$, where $T(\theta)=(\alpha+1)^{2}-4 \alpha \cos ^{2} \theta / 2$. A direct calculation certifies that the maximum of $T(\theta)$ occurs at $\theta=\pi$, and $T(\pi)=[(\alpha-1) /(\alpha+1)]^{2}>0$.

It remains to show that at $z=1,1-w=(1-z)^{2}\left[a+a_{1}(1-z)\right.$ $+\cdots], \quad \lambda \geqq 1, a>0 . \quad z=f(w)=(\alpha-1)^{2} w /(\alpha-w)^{2}$. From the equation $z=f(w)$ we obtain $1-z=(1-w)\left(\alpha^{2}-w\right) /(\alpha-w)^{2}$, which when solved for $1-w$ yields

$$
1-w=\frac{-(\alpha-1)(1-2 z-\alpha) \pm(\alpha-1)(\alpha+1) \sqrt{1-4 \alpha(1-z) /(\alpha+1)^{2}}}{-2 z} .
$$

Now divide the numerator and the denominator by -2 and write $z$ in the denominator as $1-(1-z)$.

$$
\begin{gathered}
1-w=\left\{\frac{(\alpha-1)}{2}[2(1-z)-(\alpha+1)] \pm \frac{\left(\alpha^{2}-1\right)}{-2}\left[1-\frac{4 \alpha}{2(\alpha+1)^{2}}(1-z)\right.\right. \\
\left.\left.+-\frac{1}{8} \frac{16 \alpha^{2}}{(\alpha+1)^{4}}(1-z)^{2}+\cdots\right]\right\} \cdot \sum_{k=0}^{\infty}(1-z)^{k} .
\end{gathered}
$$

Using the negative branch,

$$
\begin{aligned}
1-w= & \left\{(\alpha-1)(1-z)-\frac{\alpha\left(\alpha^{2}-1\right)}{(\alpha+1)^{2}}(1-z)-\frac{1}{8} \frac{\left(\alpha^{2}-1\right)}{2} \frac{16 \alpha^{2}}{(\alpha+1)^{4}}(1-z)^{2}\right. \\
& +\cdots\} \cdot\left\{1+(1-z)+(1-z)^{2}+\cdots\right\} . \\
= & (1-z)\left\{(\alpha-1)-\frac{\alpha(\alpha-1)}{\alpha+1}+\sum_{k=1}^{\infty} b_{k}(1-z)^{k}\right\}
\end{aligned}
$$

Theorefore $1-w=(1-z)^{\lambda}\left[a+a_{1}(1-z)+\cdots\right]$ where $\lambda=1$ and $a=(\alpha-1) /(\alpha+1)>0$.

Theorem 3, along with Theorems 1 and 2 of [2] show that the Chebyshev method is neither an $\left[F, d_{n}\right]$ nor a Sonnenschein method. 
One of the important properties of $(f, g)$-summability is the following [5, p. 267]:

Let $(f, g),\left(f_{1}, g_{1}\right)$ be two Gronwall means which map regions $D, D_{1}$ and with exponents $\lambda, \lambda_{1}$. If $\lambda>\lambda_{1}$, and $D$ is interior to $D_{1}$, then $(f, g)$ is stronger than $\left(f_{1}, g_{1}\right)$; i.e., $(f, g) \supset\left(f_{1}, g_{1}\right)$.

The de la Vallee Poussin method $(V)$ [4, p. 103] is a Gronwall method with $\delta=2^{-1}, \quad f(w)=(1-\sqrt{1-w}) /(1-\sqrt{1-w}), g(w)=$ $(1-w)^{-1 / 2}$ and $\lambda=2$.

THEOREM 4. $(V) \supset(\sigma)$.

Proof. Since $\lambda_{(V)}=2, \lambda_{(\sigma)}=1$, it is enough to show that $D(V)$ is interior to $D(\sigma)$, that is,

$$
\left|\frac{1-\sqrt{1-w}}{1+\sqrt{1-w}}\right| \leqq\left|\frac{(\alpha-1)^{2} w}{(\alpha-w)^{2}}\right| .
$$

It suffices to consider $|w|=1$; thus we need to show

$$
\frac{1}{\mid\left(1+\sqrt{1-w^{2}} \mid\right.} \leqq \frac{(\alpha-1)^{2}}{\left|(\alpha-w)^{2}\right|}
$$

Writing $1-w=\rho e^{i \phi}$, where $-\pi<\phi<\pi$, (12) becomes

$$
\left|\alpha-1+\rho e^{i \dot{\rho}}\right|^{2} \leqq(\alpha-1)^{2}\left|1+\rho^{1 / 2} e^{i \dot{\zeta} / 2}\right|^{2},
$$

i.e.,

$$
2(\alpha-1) \cos \phi+\rho \leqq 4 \alpha\left(2 \rho^{-1 / 2} \cos \phi / 2+1\right) .
$$

Since $\cos \phi / 2>0$, it is sufficient to show that $2(\alpha-1) \cos \phi+\rho \leqq 4 \alpha$, which is readily verified.

THEOREM 5. $\sigma \nsupseteq(C, 1)$.

We shall make use of the well-known result that if $A$ and $B$ are regular summability methods, and $B$ is a triangle, then $(A) \supseteqq$ $(B)$ if and only if $A B^{-1}$ is regular.

Consider $D=A C^{-1}$, where $A$ is the Chebyshev method and $C$ is $(C, 1) . \quad C^{-1}$ has entries

$$
c_{n k}^{-1}=\left\{\begin{array}{c}
-n, k=n-1 \\
n+1, k=n \\
0, \text { elsewhere } .
\end{array}\right.
$$

Then 


$$
d_{n k}= \begin{cases}(k+1) a_{n k}-(k+1) a_{n, k+1}, & k<n \\ (n+1) a_{n n}, & k=n \\ 0, & \text { elsewhere }\end{cases}
$$

We shall show that $D$ has infinite norm.

$$
\begin{aligned}
\sum_{k=0}^{n}\left|d_{n k}\right|= & \sum_{k=0}^{p-1}(k+1)\left(a_{n, k+1}-a_{n k}\right)+\sum_{k=p}^{n-1}(k+1)\left(a_{n k}-a_{n, k+1}\right) \\
& +a_{n n}(n+1) .
\end{aligned}
$$

Now,

$$
\begin{aligned}
& \begin{aligned}
\sum_{k=0}^{p-1}(k+1)\left(a_{n, k+1}-a_{n k}\right) & =\sum_{k=0}^{p-1}(k+1) a_{n, k+1}-\sum_{k=0}^{p-1} k a_{n k}-\sum_{k=0}^{p-1} a_{n k} \\
= & \sum_{j=1}^{p} j a_{n j}-\sum_{k=0}^{p-1} k a_{n k}-\sum_{k=0}^{p-1} a_{n k} \\
= & p a_{n p}-\sum_{k=0}^{p-1} a_{n k} . \\
\sum_{k=p}^{n-1}(k+1)\left(a_{n k}\right. & \left.-a_{n, k+1}\right)=\sum_{k=p}^{n-1} k a_{n k}+\sum_{k=p}^{n-1} a_{n k}-\sum_{k=p}^{n-1}(k+1) a_{n, k+1} \\
= & \sum_{k=p}^{n-1} k a_{n k}+\sum_{k=p}^{n-1} a_{n k}-\sum_{j=p+1}^{n} j a_{n j} \\
= & p a_{n p}-n a_{n n}+\sum_{k=p}^{n-1} a_{n k} .
\end{aligned}
\end{aligned}
$$

Therefore,

$$
\sum_{k=0}^{n}\left|d_{n k}\right|=p a_{n p}-\sum_{k=0}^{p-1} a_{n k}+p a_{n p}-n a_{n n}+\sum_{k=p}^{n-1} a_{n k}+a_{n n}(n+1) .
$$

Since the Chebyshev method has row sums equal to 1 ,

$$
\sum_{k=p}^{n-1} a_{n k}=1-\sum_{k=0}^{p-1} a_{n k}-a_{n n}
$$

Thus

$$
\sum_{k=0}^{n} d_{n k}=2 p a_{n p}-2 \sum_{k=0}^{p-1} a_{n k}+1
$$

But $\sum_{k=0}^{p-1} a_{n k} \leqq 1$, so it is sufficient to show $p a_{n p} \rightarrow \infty$. This follows immediately from (2), since $\lim \sqrt{n}=\infty$ and the remaining limits have already been shown to be finite and nonzero.

The Fourier series

$$
\sum_{k=1}^{\infty} \sin k t / k=(\pi-t) / 2, \quad 0<t \leqq \pi
$$

converges for all $t$, and the function has a jump at $t=0$. Hence 
the convergence is nonuniform at $t=0$; that is, the sequence $\left\{s_{n}\left(t_{n}\right)\right\}$, where $\left\{t_{n}\right\}$ is a positive null sequence and

$$
s_{n}(t)=\sum_{k=1}^{n} \sin k t / k, \quad s_{0}=0,
$$

has several limit points, depending on the manner in which $t_{n}$ approaches 0 .

If $\lim n t_{n}=\tau \geqq 0$, then $\lim s_{n}\left(t_{n}\right)=\int_{0}^{\tau}(\sin t / t) d t$, and the maximal limit is attained when $\tau=\pi$, in which case

$$
\lim s_{n}\left(t_{n}\right)=\int_{0}^{\pi} \frac{\sin t}{t} d t=\frac{\pi}{2} \times 1.17897 \cdots .
$$

On the other hand, $(\pi-t) / 2 \rightarrow \pi / 2$ as $t \downarrow 0$. Thus the limit points of $\left\{s_{n}\left(t_{n}\right)\right\}$ cover an interval which extends beyond $f(0+)$ if $f(0+) \neq 0$. This situation is called the Gibbs phenomenon relative to the partial sums.

We shall now show that the corresponding phenomenon occurs for the Chebyshev means.

THeOREM 6. The Chebyshev means of (13) satisfy

$$
\lim \sigma_{n}\left(t_{n}\right)=\int_{0}^{\tau / \sqrt{2}} \frac{\sin y}{y} d y \text { as } n t_{n} \longrightarrow \tau \text { and } n t_{n}^{2} \longrightarrow 0,
$$

and

$$
\lim \sup \sigma_{n}\left(t_{n}\right) \leqq \int_{0}^{\pi} \frac{\sin t}{t} d t
$$

The lim sup inequality is an immediate consequence of (14) and the well-known fact that, for any totally regular matrix $A$, and any sequence $x=\left\{x_{n}\right\}, \lim \sup A_{n}(x) \leqq \lim \sup x_{n}$.

The proof of the theorem is similar to that of [6]. One may write $s_{n}(t)$ in the form

$$
s_{n}(t)=-t / 2+\int_{0}^{t} \frac{\sin (n+1 / 2) x}{2 \sin (x / 2)} d x .
$$

Since $\sin (k+1 / 2) x=\mathscr{J}(\exp (i(k+1 / 2) x))$,

$$
\sigma_{n}(t)=-t / 2+\mathscr{J}\left[\int_{0}^{t} \frac{1}{2 \sin (x / 2)} \sum_{k=0}^{n} a_{n k} e^{i k x} e^{i x / 2} d x\right] .
$$

From [1, p. 297], $\quad \sum_{k=0}^{n} a_{n k} e^{i k x}=T_{n}\left(1+2 e^{i x}\right) / T_{n}(3)$, where $T_{n}(x) \quad$ is defined by (1).

Define 


$$
\begin{aligned}
\rho e^{i \beta} & =1+2 e^{i x}+\left[\left(1+2 e^{i x}\right)^{2}-1\right]^{1 / 2} \\
& =1+2 e^{i x}+2 e^{i x / 2} e^{i x / 4}(2 \cos x / 2)^{1 / 2} .
\end{aligned}
$$

Let $a=(2 \cos x / 2)^{1 / 2}$. Then $\rho \cos \beta=1+2(\cos x+\alpha \cos (3 x / 4))$,

$$
\rho \sin \beta=2(\sin x+a \sin (3 x / 4)),
$$

and

$$
\rho^{2}=5+4(\cos x+a \cos (3 x / 4))+8(\cos (x / 2)+a \cos (x / 4)) .
$$

Therefore $1+2 e^{i x}-\left[\left(1+2 e^{i x}\right)^{2}-1\right]^{1 / 2}=\rho^{-1} e^{-i \beta}$, and assume $0<x \leqq$ $t \leqq \pi / 2$.

$$
\begin{aligned}
\sigma_{n}(t) & +t / 2=\frac{1}{2 T_{n}(3)} \int_{0}^{t} \frac{1}{2 \sin (x / 2)}\left[\rho^{n} \sin (n \beta+x / 2)\right. \\
& \left.-\rho^{-n} \sin (n \beta-x / 2)\right] d x=\frac{1}{4 T_{n}(3)}\left\{\int_{0}^{t} \rho^{n} \cot (x / 2) \sin n \beta d x\right. \\
& \left.+\int_{0}^{t} \rho^{n} \cos n \beta d x+-\int_{0}^{t} \rho^{-n} \cot (x / 2) \sin n \beta d x+\int_{0}^{t} \rho^{-n} \cos n \beta d x\right\} .
\end{aligned}
$$

From (17), $\rho$ is monotone decreasing in $x$ for $0<x \leqq \pi / 2$. Therefore for $0<x \leqq \pi / 2, \rho<\alpha$. Thus

$$
\left|\frac{1}{2 T_{n}(3)} \int_{0}^{t} \rho^{n} \cos n \beta d x\right|<\int_{0}^{t}(\rho / \alpha)^{n} d x<t,
$$

so that there exists an $\eta$ satisfying $|\eta|<1$ such that

$$
\frac{1}{2 T_{n}(3)} \int_{0}^{t} \rho^{n} \cos n \beta d x=\eta t \text {. }
$$

Now assume that $t=t_{n}, n t_{n} \rightarrow \tau, 0 \leqq \tau \leqq \infty$, and $n t_{n}^{2} \rightarrow 0$.

Since, from (17), $\rho \geqq \sqrt{5}$,

$$
\left|\frac{1}{4 T_{n}(3)} \int_{0}^{t} \rho^{-n} \cos n \beta d x\right|<\pi / 4\left(\alpha \sqrt{5)^{n}}=o(1) .\right.
$$

$$
\left.\mid \frac{1}{4 T_{n}(3)} \int_{0}^{t} \rho^{-n} \cot (x / 2) \sin n \beta d x\right) \mid<\frac{1}{2\left(\alpha \sqrt{5)^{n}}\right.} \int_{0}^{t} n \beta \cot (x / 2) d x .
$$

We wish to show that $\beta<x$. For $0<x \leqq \pi / 2$, from (16), $\rho \sin \beta<2(1+a) \sin x$. From (17), if $\cos (3 x / 4)+2 \cos (x / 4) \geqq 2$, then $\rho>2(a+1)$. In the interval $[0, \pi / 2]$,

$$
\begin{aligned}
\cos (3 x / 4) & +2 \cos (x / 4) \geqq \cos (3 \pi / 8)+\cos (\pi / 8) \\
& =\cos (\pi / 8)\left(4 \cos ^{2}(\pi / 8)-1\right) .
\end{aligned}
$$

Since $\cos (\pi / 8)=\sqrt{2+\sqrt{2}} / 2$, it is sufficient to show that 


$$
\frac{\sqrt{2+\sqrt{2}}}{2}\left(\frac{4}{4}(2+\sqrt{2})-1\right) \geqq 2,
$$

which is easily verified. Therefore $0<\sin <\beta(\rho / 2(1+a)) \sin \beta<$ $\sin x$, and $\beta<x$.

For $0<x \leqq \pi / 2, \quad 2 \leqq x / \sin (x / 2) \leqq \pi / \sqrt{2}$. Substituting in (18) we have

$$
\begin{gathered}
\left|\frac{1}{4 T_{n}(3)} \int_{0}^{t} \rho^{-n} \cot (x / 2) \sin n \beta d x\right|<\frac{n}{2\left(\alpha \sqrt{5)^{n}}\right.} \int_{0}^{\pi / 2} \cos (x / 2) \cdot \frac{x}{\sin (x / 2)} d x \\
<\frac{n \pi^{2}}{4 \sqrt{2}(\alpha \sqrt{5})^{n}}=o(1),
\end{gathered}
$$

and

$$
\sigma_{n}(t)+(1-\eta) t / 2=\frac{1}{4 T_{n}(3)} \int_{0}^{t} \rho^{n} \cot (x / 2) \sin n \beta d x+o(1) .
$$

Using (17), and the values of $\alpha$ and $\alpha$,

$$
\begin{gathered}
1-(\rho / \alpha)^{2}=[17+12 \sqrt{2}-5-4(\cos x+a \cos (3 x / 4)) \\
-8(\cos (x / 2)+a \cos (x / 4))] / \alpha^{2} \\
=\frac{4}{\alpha^{2}}[1-\cos x+2(1-\cos (x / 2))+\sqrt{2}(1-\cos (3 x / 4) \sqrt{\cos (x / 2})) \\
+2 \sqrt{2(1}-\cos (x / 4) \sqrt{\cos (x / 2))}] .
\end{gathered}
$$

Since $0<\cos (x / 2)<1$,

$$
\begin{gathered}
1-\cos (x / 4) \sqrt{\cos (x / 2)} \leqq 1-\cos (x / 4) \cos (x / 2) \\
=1-(\cos (3 x / 4)+\cos (x / 4)) / 2 .
\end{gathered}
$$

Similarly, $1-\cos (3 x / 4) \sqrt{\cos (x / 2)} \leqq 1-(\cos (5 x / 4)+\cos (x / 4)) / 2$. Therefore,

$$
\begin{aligned}
& 1-(\rho / \alpha)^{2} \leqq \frac{4}{\alpha^{2}}\left[2 \sin ^{2}(x / 2)+4 \sin ^{2}(x / 4)+\sqrt{2(2} \sin ^{2}(5 x / 8)\right. \\
& \left.\left.+2 \sin ^{2}(x / 8)\right) / 2+\sqrt{2}\left(2 \sin ^{2}(3 x / 8)+2 \sin ^{2}(x / 8)\right)\right] \\
& \leqq \frac{4}{\alpha^{2}}\left[2(x / 2)^{2}+4(x / 4)^{2}+\sqrt{2}\left((5 x / 8)^{2}+(x / 8)^{2}\right)\right. \\
& \left.\left.+2 \sqrt{2\left((3 x / 8)^{2}\right.}+(x / 8)^{2}\right)\right] \\
& =\frac{4}{\alpha^{2}}\left(3 / 4+\frac{46 \sqrt{2}}{64}\right) x^{2}<\frac{x^{2}}{4} \text {. }
\end{aligned}
$$

Since $0<\rho / \alpha<1,1-\rho / \alpha \leqq 1-(\rho / \alpha)^{2}$, so that $1-\rho / \alpha<x^{2} / 4$. $0<$ 
$1-(\rho / \alpha)^{n}=(1-\rho / \alpha) \sum_{k=0}^{n-1}(\rho / \alpha)^{k}<n(1-\rho / \alpha)<n x^{2} / 4$. Therefore 1$(\rho / \alpha)^{n}=\lambda n x^{2}$ for some $\lambda$ satisfying $0<\lambda<1 / 4$, so that we may write

$$
\begin{aligned}
\frac{1}{2 T_{n}(3)} \int_{0}^{t} \rho^{n} \cot & (x / 2) \sin n \beta d x=\frac{\alpha^{n}}{2 T_{n}(3)}\left[\int_{0}^{t} \cot (x / 2) \sin n \beta d x\right. \\
& \left.-n \int_{0}^{t} \lambda x^{2} \cot (x / 2) \sin n \beta d x\right] \\
& \times n\left|\int_{0}^{t} \lambda x^{2} \cot (x / 2) \sin n \beta d x\right|<n \int_{0}^{t} x^{2} \cot (x / 2) d x \\
\leqq & \frac{n t \pi}{\sqrt{2}} \int_{0}^{t} d x<n t^{2}=o(1),
\end{aligned}
$$

since $\lim n t_{n}^{2}=0 . \quad$ Note that $\lim \alpha^{n} / 2 T_{n}(3)=1$.

Using (17),

$$
\begin{gathered}
\frac{\rho \beta}{2}-\frac{2}{\alpha}\left(1+\frac{3 \sqrt{2}}{4}\right) x=\frac{\rho}{\alpha}(\beta-\sin \beta)-\frac{2}{\alpha}(x-\sin x) \\
-\frac{2 \sqrt{2}}{\alpha}\left(\frac{3 x}{4}-\sin (3 x / 4) \sqrt{\cos (x / 2)}\right)
\end{gathered}
$$

so that

$$
\begin{aligned}
|\rho \beta / \alpha-r x| & \leqq \frac{\rho}{\alpha}|\beta-\sin \beta|+\frac{2}{\alpha}|x-\sin x| \\
+ & \frac{2 \sqrt{2}}{\alpha}\left|\frac{3 x}{4}-\sin (3 x / 4) \sqrt{\cos (x / 2)}\right|,
\end{aligned}
$$

where $r=2(1+3 \sqrt{2 / 4}) / \alpha=(4+3 \sqrt{2}) / 2 \alpha=(4+3 \sqrt{2})(3-2 \sqrt{2}) / 2=$ $1 / \sqrt{2}$.

But $\quad 0 \leqq 3 x / 4-\sin (3 x / 4) \cdot \sqrt{\cos (x / 2)} \leqq 3 x / 4-\sin (3 x / 4) \cos (x / 2)$, $\sin (3 x / 4) \geqq 3 x / 4-(3 x / 4)^{3} / 3$ ! , and $\cos (x / 2) \geqq 1-x^{2} / 4$, so that

$$
\begin{gathered}
\mid 3 x / 4-\sin (3 x / 4) \sqrt{\cos (x / 2) \mid} \leqq 3 x / 4-\left(3 x / 4-(3 x / 4)^{3} / 6\right)\left(1-x^{2} / 4\right) \\
=33 x^{3} / 128 .
\end{gathered}
$$

Since $0<x-\sin x<x^{3}$ and $\beta<x$,

$$
|\rho \beta / \alpha-x / \sqrt{2}| \leqq\left(\rho \beta^{3}+2 x^{3}+33 \sqrt{2} x^{3} / 64\right) / \alpha<2 x^{3} .
$$

Also, $|\beta-x / \sqrt{2}| \leqq \mid \rho \beta / \alpha-x / \sqrt{2 \mid}+(1-\rho / \alpha) \beta<2 x^{3}+x^{3}=x^{3}$, so that $\beta=x / \sqrt{2}+\mu x^{3}$, where $|\mu|<3$.

The remainder of the proof of (15) is the same as that of [6], beginning with formula (2.7), and will therefore be omitted. 


\section{REFERENCES}

1. G. G. Bilodeau, On the use of summability methods to increase the rate of convergence of series, J. Math. and Physics, 40 (1961), 289-299.

2. J. Bustoz and D. Wright, A Note on Gronwall Transforms, J. Applicable Analysis, 6 (1976), 39-46.

3. R. G. Cooke, Infinite Matrices and Sequence Spaces, Macmillan and Co. Ltd., 1950.

4. T. H. Gronwall, Summation of series and conformal mapping, Annals of Math., 32 (1932), 101-117.

5. W. T. Scott and H. S. Wall, The transformation of series and sequences, Trans. Amer. Math. Soc., 51 (1942), 255-279,

6. O. Szasz, On the Gibbs' phenomenon for Euler means, Acta Sci. Math., 12 (1950), 107-111.

Received August 25, 1977 and in revised form July 12, 1978.

UNIVERSITY OF WISCONSIN-STEVENS PoINT

Stevens PoInt, WI 54481

AND

INDIANA UNIVERSITY

BLOOMINGTON, IN 47405 



\section{PACIFIC JOURNAL OF MATHEMATICS}

\section{EDITORS}

RICHARD ARENS (Managing Editor)

University of California

Los Angeles, California 90024

C. W. CURTIS

University of Oregon

Eugene, OR 97403

C. C. MOORE

University of California

Berkeley, CA 94720
J. DugundjI

Department of Mathematics University of Southern Californı Los Angeles, California 90007

R. Finn and J. Milgram Stanford University Stanford, California 94305

ASSOCIATE EDITORS

E. F. BECKENBACH

B. H. NeUmanN

F. WOLF

K. YoSHIDA

\section{SUPPORTING INSTITUTIONS}

UNIVERSITY OF BRITISH COLUMBIA CALIFORNIA INSTITUTE OF TECHNOLOGY

UNIVERSITY OF CALIFORNIA

MONTANA STATE UNIVERSITY

UNIVERSITY OF NEVADA, RENO

NEW MEXICO STATE UNIVERSITY

OREGON STATE UNIVERSITY

UNIVERSITY OF OREGON
UNIVERSITY OF SOUTHERN CALIFORNIA

STANFORD UNIVERSITY

UNIVERSITY OF HAWAII

UNIVERSITY OF TOKYO

UNIVERSITY OF UTAH

WASHINGTON STATE UNIVERSITY

UNIVERSITY OF WASHINGTON 


\section{Pacific Journal of Mathematics}

\section{Vol. 80, No. $1 \quad$ September, 1979}

Jeroen Bruijning and Jun-iti Nagata, A characterization of covering dimension by

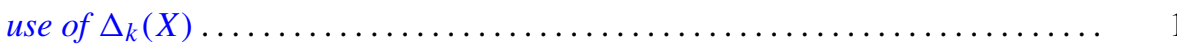

John J. Buoni and Albert Jonathan Klein, On the generalized Calkin algebra ...... 9

Thomas Ashland Chapman, Homotopy conditions which detect simple homotopy

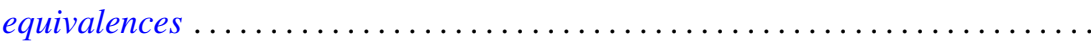

John Albert Chatfield, Solution for an integral equation with continuous interval

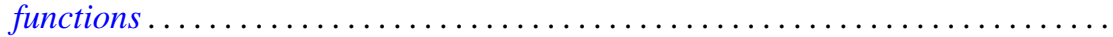

Ajit Kaur Chilana and Ajay Kumar, Spectral synthesis in Segal algebras on

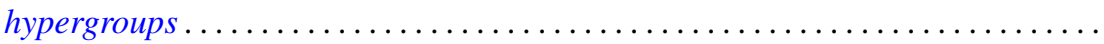

Lung O. Chung, Jiang Luh and Anthony N. Richoux, Derivations and

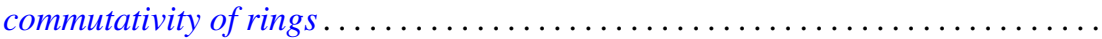

Michael George Cowling and Paul Rodway, Restrictions of certain function spaces to closed subgroups of locally compact groups .....................

David Dixon, The fundamental divisor of normal double points of surfaces........

Hans Georg Feichtinger, Colin C. Graham and Eric Howard Lakien,

Nonfactorization in commutative, weakly selfadjoint Banach algebras . . . . . . .

Michael Freedman, Cancelling 1-handles and some topological imbeddings ....... .

Frank E., III Gerth, The Iwasawa invariant $\mu$ for quadratic fields . . . . . . . . . . . . . .

Maurice Gilmore, Three-dimensional open books constructed from the identity

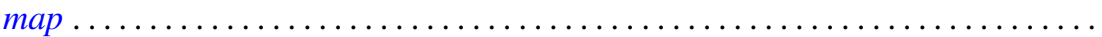

Stanley P. Gudder, A Radon-Nikodým theorem for $*$-algebras .

Peter Wamer Harley, III and George Frank McNulty, When is a point Borel? .

Charles Henry Heiberg, Fourier series with bounded convolution powers . .

Rebecca A. Herb, Characters of averaged discrete series on semisimple real Lie

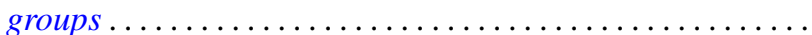

Hideo Imai, On singular indices of rotation free densities . .

Sushil Jajodia, On 2-dimensional CW-complexes with a single 2-cell . . .

Herbert Meyer Kamowitz, Compact operators of the form $u C_{\varphi}$

Matthew Liu and Billy E. Rhoades, Some properties of the Chebyshev method...

213

George Edgar Parker, Semigroups of continuous transformations and generating

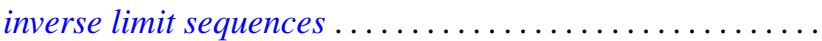

Samuel Murray Rankin, III, Oscillation results for a nonhomogeneous

equation ...

Martin Scharlemann, Transverse Whitehead triangulations ...

Gary Joseph Sherman, A lower bound for the number of conjugacy classes in a

finite nilpotent group

Richard Arthur Shoop, The Lebesgue constants for $\left(f, d_{n}\right)$-summability .

Stuart Jay Sidney, Functions which operate on the real part of a uniform

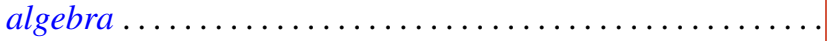

Tim Eden Traynor, The group-valued Lebesgue decomposition 